\title{
Cardiology and Cardiovascular Medicine
}

Volume 1, Issue 1

Image Article

\section{Resolution of Inferior Wall Ischemia after Successful Revascularization of LAD Lesion: The Value of Myocardial Perfusion Imaging in Guiding Management of Multi-vessel CAD}

\author{
Chien-Cheng Chen ${ }^{1}$, Ya-Lei Niu², Guang-Uei Hung ${ }^{3,4,5^{*}}$ \\ ${ }^{1}$ Section of Cardiology, Department of Internal Medicine, Show Chwan Memorial Hospital, Changhua, Taiwan \\ ${ }^{2}$ Section of Cardiology, Department of Internal Medicine, Chang Bing Show Chwan Memorial Hospital, Changhua, \\ Taiwan \\ ${ }^{3}$ Department of Nuclear Medicine, Chang Bing Show Chwan Memorial Hospital, Changhua, Taiwan \\ ${ }^{4}$ Department of Biomedical Imaging and Radiological Science, China Medical University, Taichung, Taiwan \\ ${ }^{5}$ Department of Medical Imaging and Radiological Science, Central Taiwan University of Science and Technology, \\ Taichung, Taiwan
}

*Corresponding Author: Guang-Uei Hung, M.D., Department of Nuclear Medicine, Chang Bing Show Chwan Hospital, 6 Lukon Road, Lukong Town, Changhua Shien, Taiwan 505, Taiwan. E-mail: 106143@gmail.com

Received: 25 January 2017; Accepted: 03 February 2017; Published: 07 February 2017

\begin{abstract}
A 53-year-old male with history of hypertension and hyperlipidemia was presented with angina pectoris. Multi-slice CT coronary angiography showed severe stenosis in both left anterior descending coronary artery (LAD) and right coronary artery (RCA). Myocardial perfusion imaging (MPI) revealed extensive ischemia in apex, anterior, septal and inferior walls. Invasive coronary angiography revealed total occlusion in RCA and an $80 \%$ stenosis in LAD. Revascularization was performed for LAD first due to much severer ischemia than RCA on MPI. Follow-up MPI showed resolution of ischemia in both LAD and RCA territories, and the patient remained symptom-free during two years of follow-up.
\end{abstract}



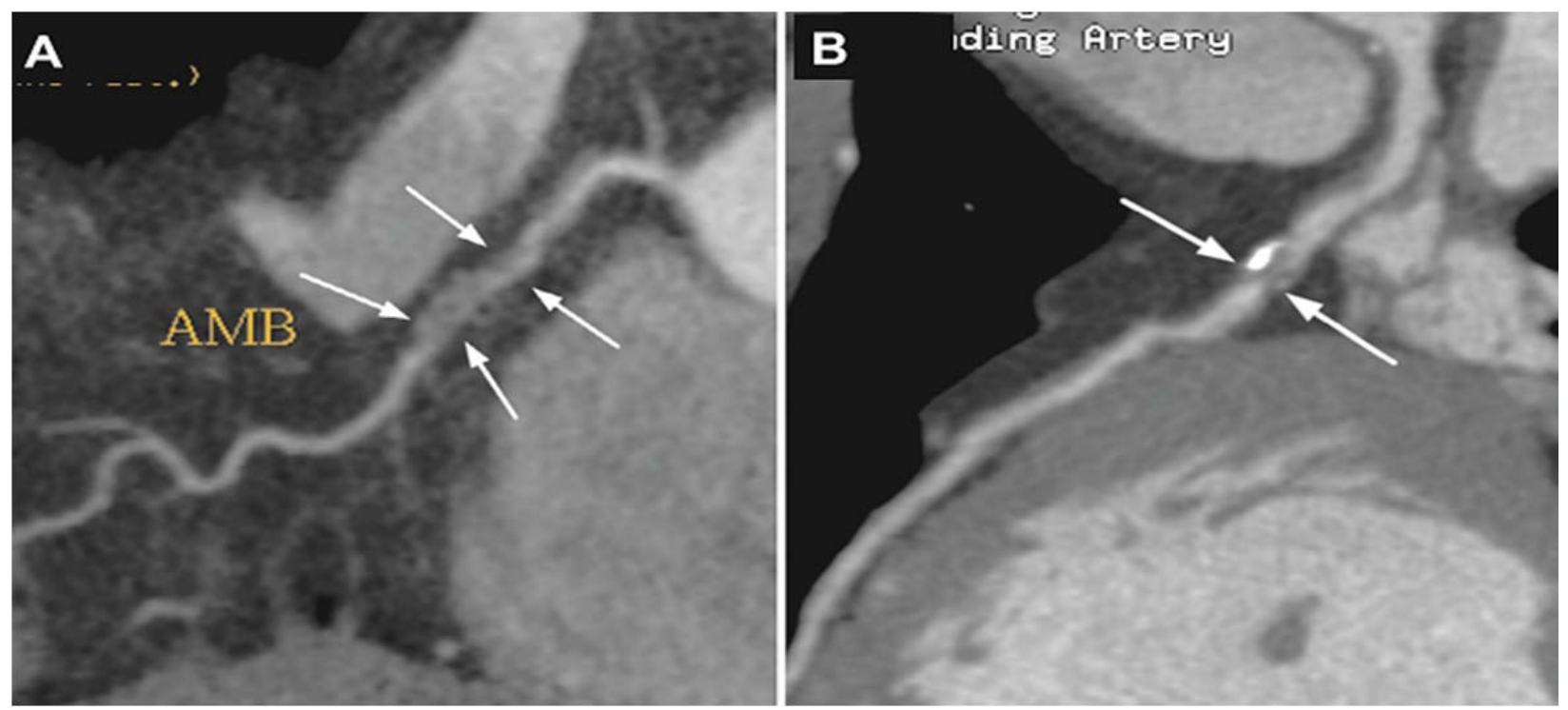

Figure 1: A 53-year-old man had past history of hypertension and hyperlipidemia for years without regular control. He complained of exertional chest tightness and dyspnea for two months. Sixty-four slices cardiac computed tomography (CT) was performed as the initial non-invasive imaging work-up for possible coronary artery disease (CAD). The coronary calcium scoring was 70, 60 and 0 for left anterior descending artery (LAD), left circumflex artery (LCX) and right coronary artery (RCA), respectively [1]. CT coronary angiography (CTA) revealed: (A) noncalcified plaques at proximal RCA with nearly total occlusion (arrows); and (B) mixed calcified and non-calcified plaques at proximal LAD also with nearly total occlusion (arrows).

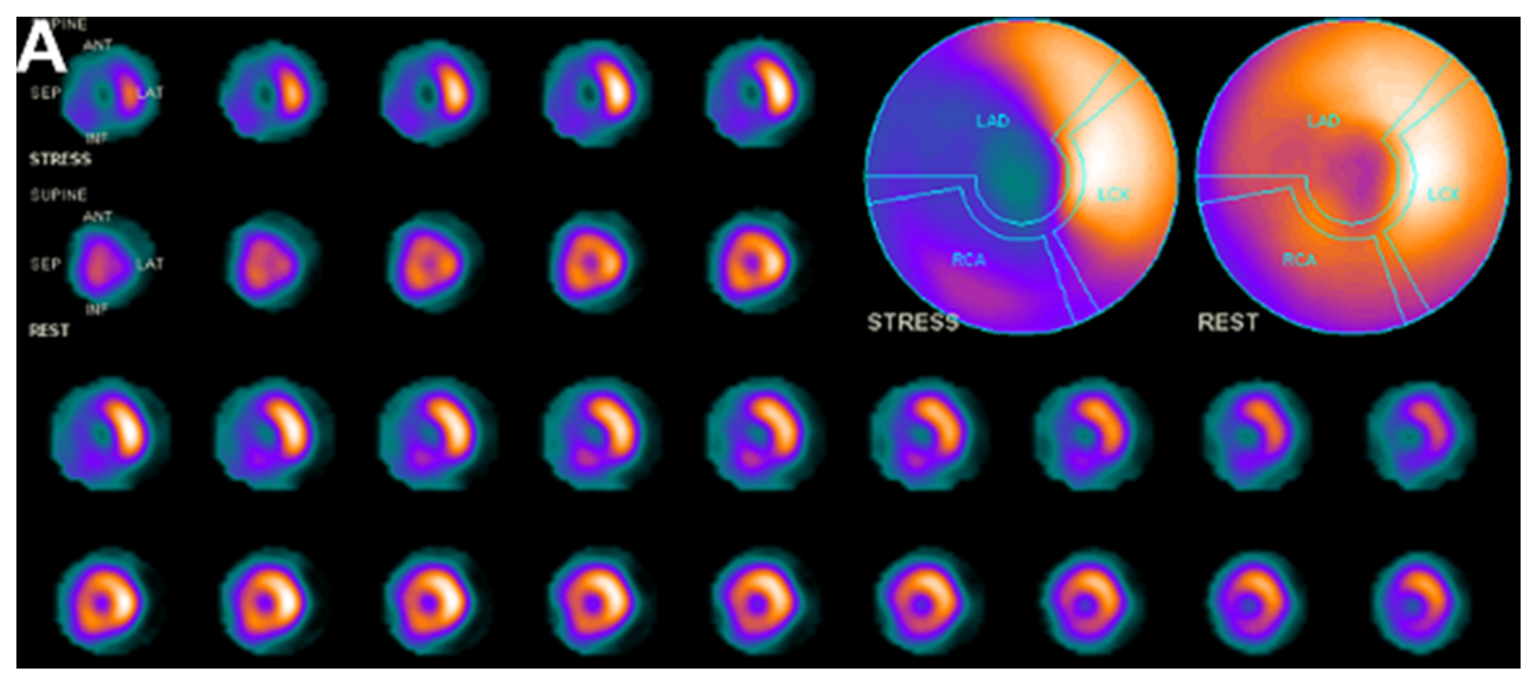

Figure 2: For evaluating the physiological significance of these two coronary lesions on CTA, dipyridam1olestress rest Tl-201 myocardial perfusion imaging (MPI) was further arranged for him [2]. Figure 2 (A): The perfusion images revealed moderate to severe grade of extensive ischemia involving apex, anterior wall, septum and 
inferior wall [3]. The ischemic \% myocardium was 30\% and 10\% for LAD and RCA territories, respectively [4, 5]. The transient ischemic dilation (TID) ratio was 1.76 [6].

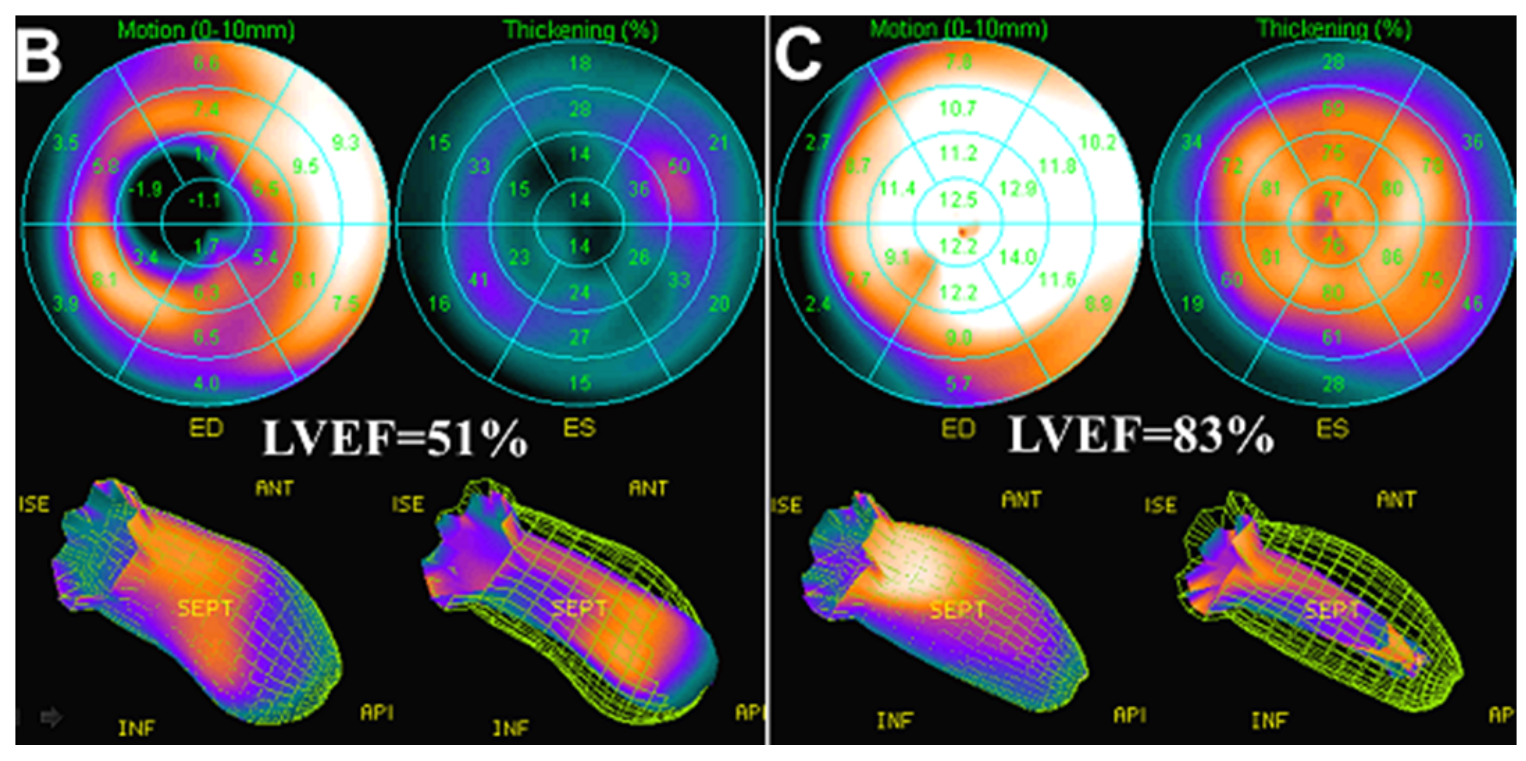

Figures 2 (B and C): The functional analysis of MPI revealed that the early post-stress (B) and rest (C) ejection fraction of left ventricle were $51 \%$ and $83 \%$, respectively [7]. Besides, severe hypokinesis in LAD territory was noted on the early post-stress wall motion analysis (B) but returned to normokinesis on rest (C), indicating of stressinduced stunning. All these findings were high-risk indicators of cardiac events; therefore, the patient was further referred for invasive coronary angiography [8].

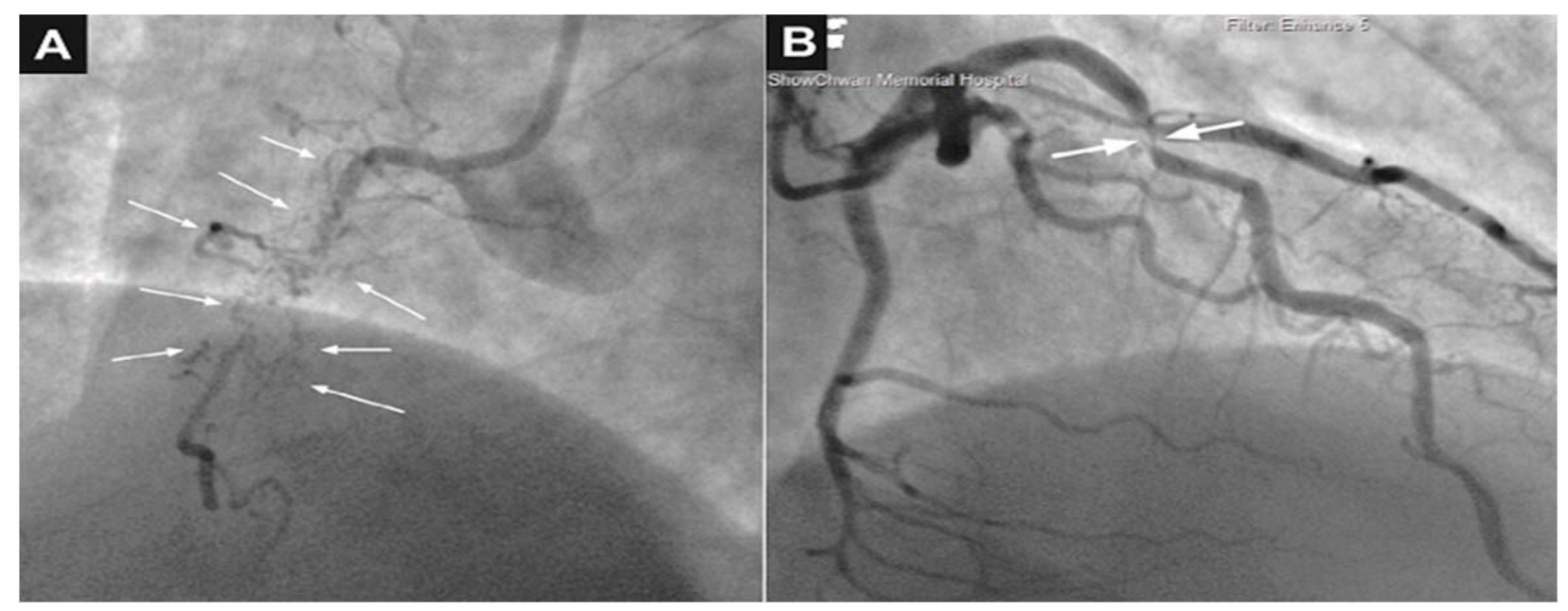

Figure 3: Subsequent coronary angiography revealed: (A) chronic total occlusion (CTO) in proximal portion of RCA and (B) an 80\% stenosis in proximal portion of LAD. Although severer stenosis was noted in RCA than LAD, Cardiol Cardiovascmed 2017; 1 (1): 58-62 
percutaneous coronary intervention (PCI) was performed for the LAD lesion first because much severer ischemia was noted in LAD territory than in RCA.

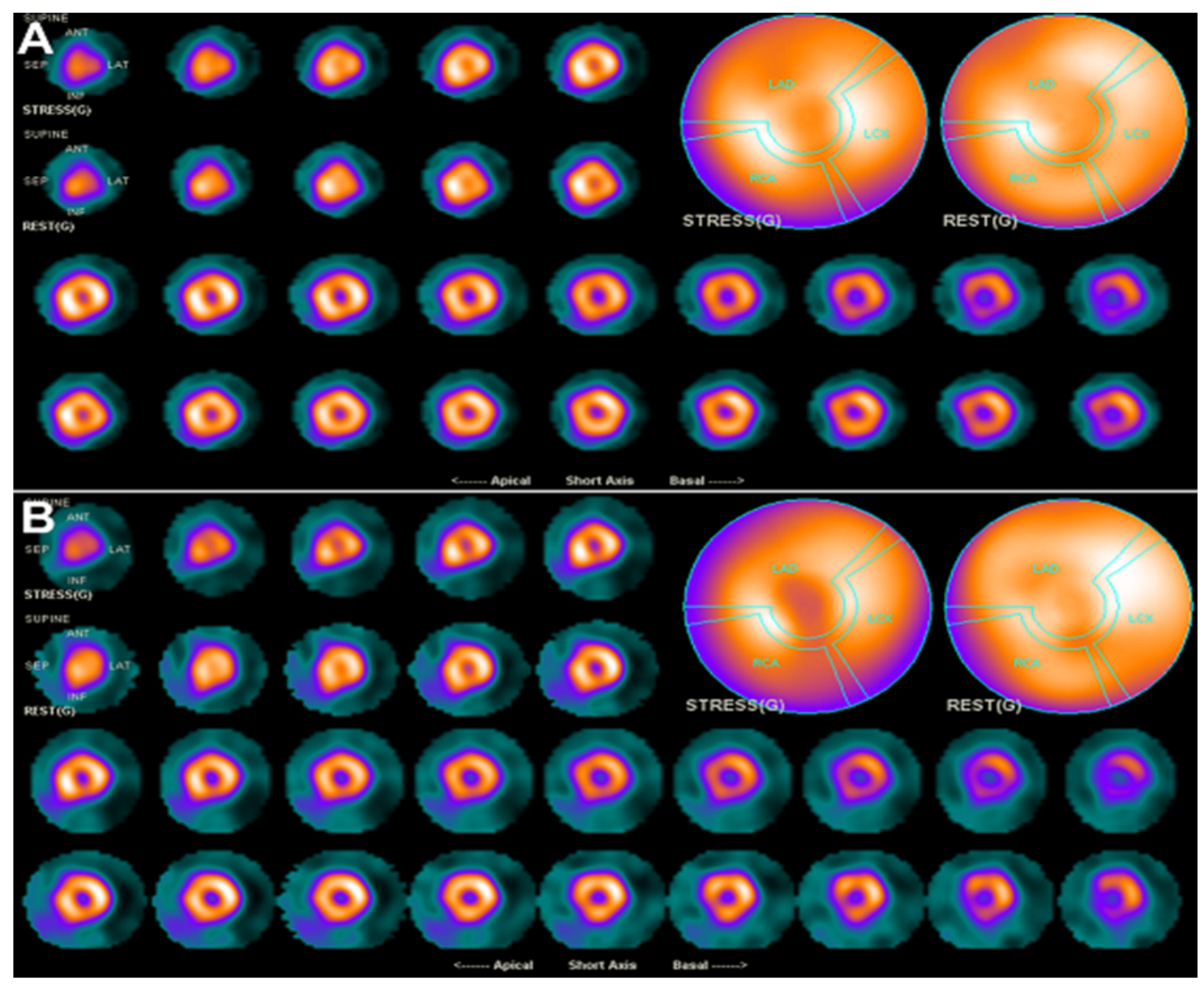

Figure 4: Shortly after successful revascularization of the LAD lesion, the patient's chest discomforts disappeared quickly. (A) Follow-up MPI was performed 5 months after PCI for checking residual ischemia from the CTO lesion of RCA [9-11]. Surprisingly, it showed near-total resolution of ischemia in not only the LAD but also RCA territories, with minimally residual ischemia in basal inferior and basal inferoseptal segments (about $2 \%$ ischemic myocardium). The resolution of ischemia in RCA territory after successful revascularization of LAD lesion was considered related to well-development of collateral blood supply from LAD to RCA. (B) A second follow-up MPI performed 2 years after PCI revealed very similar picture as 1st follow-up MPI and the patient remains satisfied with the control of angina symptom. The presented case reminded us again that MPI provided the information regarding physiological significance of coronary lesions in patients with CAD and was very useful in the treatment planning of multi-vessel CAD for obtaining a favorable outcome.

\section{Conflict of Interest}

The authors declare that they have no conflict of interest. 


\section{References}

1. Fujimura T, Miura T, Nao T, Yoshimura M, Nakashima Y, et al. Dual-source computed tomography coronary angiography in patients with high heart rate. Heart Vessels 29 (2014): 443-448.

2. Schuijf JD, Wijns W, Jukema JW, Atsma DE, de Roos A, et al. Relationship between noninvasive coronary angiography with multi-slice computed tomography and myocardial perfusion imaging. J Am Coll Cardiol 48 (2006): 2508-2514.

3. Hachamovitch R, Hayes SW, Friedman JD, Cohen I, Berman DS. Comparison of the short-term survival benefit associated with revascularization compared with medical therapy in patients with no prior coronary artery disease undergoing stress myocardial perfusion single photon emission computed tomography. Circulation 17(2003): 2900-2907.

4. Gaemperli O, Schepis T, Valenta I, Husmann L, Scheffel H, et al. Cardiac image fusion from stand-alone SPECT and CT: clinical experience. J Nucl Med 48 (2007): 696-703.

5. Javadi MS, Lautamäki R, Merrill J, Voicu C, Epley W, et al. Definition of vascular territories on myocardial perfusion images by integration with true coronary anatomy: a hybrid PET/CT analysis. J Nucl Med 51 (2010): 198-203.

6. Mazzanti M, Germano G, Kiat H, Kavanagh PB, Alexanderson E, et al. Identification of severe and extensive coronary artery disease by automatic measurement of transient ischemic dilation of the left ventricle in dualisotope myocardial perfusion SPECT. J Am Coll Cardiol 27 (1996): 1612-1620.

7. Hung GU, Lee KW, Chen CP, Yang KT, Lin WY. Worsening of left ventricular ejection fraction induced by dipyridamole on Tl-201 gated myocardial perfusion imaging predicts significant coronary artery disease. J Nucl Cardiol 13 (2006): 225-232.

8. Berman DS, Shaw LJ, Hachamovitch R, Friedman JD, Polk DM, et al. Comparative use of radionuclide stress testing, coronary artery calcium scanning, and noninvasive coronary angiography for diagnostic and prognostic cardiac assessment. Semin Nucl Med 37 (2007): 2-16.

9. Shah PB. Management of coronary chronic total occlusion. Circulation 123 (2011): 1780-1784.

10. Arai T, Kawamura A, Yuasa S, Maekawa Y, Fukuda K. Successful coronary intervention of chronic total occlusion of the right coronary artery by ipsilateral injection via an isolated conus artery. Heart Vessels 27 (2012): 327-330.

11. Dai J, Katoh O, Zhou H, Kyo E. First reported revascularization of complex occlusion of the right coronary artery using the IVUS-guided reverse CART technique via a gastroepiploic artery graft. Heart Vessels 31 (2016): 251-255.

This article is an open access article distributed under the terms and conditions of the Creative Commons Attribution (CC-BY) license 4.0 